\title{
CONDITIONALLY COMPACT SEMITOPOLOGICAL ONE-PARAMETER INVERSE SEMIGROUPS OF PARTIAL ISOMETRIES
}

\author{
M. O. BERTMAN
}

\begin{abstract}
The algebraic structure of one-parameter inverse semigroups has been completely described. Furthermore, if $B$ is the bicyclic semigroup and if $B$ is contained in any semitopological semigroup, the relative topology on $B$ is discrete. We show that if $F$ is an inverse semigroup generated by an element and its inverse, and $F$ is contained in a compact semitopological semigroup, then the relative topology is discrete; in fact, if $F$ is any one-parameter inverse semigroup contained in a compact semitopological semigroup, then the multiplication on $F$ is jointly continuous if and only if the inversion is continuous on $F$, and we describe $\bar{F}$ in that case. We also show that if $\left\{J_{t}\right\}$ is a one-parameter semigroup of bounded linear operators on a (separable) Hilbert space, then $\left\{J_{t}\right\} \cup\left\{J_{t}^{*}\right\}$ generates a one-parameter inverse semigroup $T$ with $J_{t}^{-1}=J_{t}^{*}$ if and only if $\left\{J_{t}\right\}$ is a one-parameter semigroup of partial isometries, and we describe the weak operator closure of $T$ in that case.
\end{abstract}

1. Introduction. An inverse semigroup is a semigroup in which each element $x$ has a unique inverse $x^{-1}$ with the properties that $x x^{-1} x=x$ and $x^{-1} x x^{-1}$ $=x^{-1}$. If $G$ is any subgroup of the positive real numbers under ordinary multiplication, let $P=G \cap[1, \infty)$. Let $F_{P}=\left\{(x, y, z) \in P^{3}: y>x\right.$ and $y>z\}$ together with the following multiplication:

$$
(x, y, z)(r, s, t)=\left(\frac{x z r}{y \wedge z r}, \frac{y z r s}{(y \wedge z r)(z r \wedge s)}, \frac{z r t}{z r \wedge s}\right)
$$

where $x \wedge y=\min (x, y)$. Any inverse semigroup generated by a homomorphic image of $P$ is a homomorphic image of $F_{P}[6]$ and is called a one-parameter inverse semigroup. We mention at this point one homomorphic image of $F_{P}$. Let $B_{P}=P \times P$ together with this multiplication:

$$
(x, y)(z, w)=\left(\frac{x z}{y \wedge z}, \frac{y w}{y \wedge z}\right) .
$$

Received by the editors March 1, 1976.

AMS (MOS) subject classifications (1970). Primary 47D05, 22A10.

Key words and phrases. Partial isometries, one-parameter semigroups, inverse semigroups, compact semitopological semigroups. 
If $P=\left\{1, x, x^{2}, \ldots\right\}$ where $x>1$, then $B_{P}$ is the bicyclic semigroup whose properties are discussed in [4].

Suppose that $Y$ is a semigroup with a Hausdorff topology in which the maps $x \rightarrow x y$ and $x \rightarrow y x$ of $Y \rightarrow Y$ are continuous for each $y$ in $Y$. Then the multiplication on $Y$ is said to be separately continuous and $Y$ is called a semitopological semigroup. If the map $(x, y) \rightarrow x y$ of $Y \times Y \rightarrow Y$ is continuous, then the multiplication on $Y$ is jointly continuous (or just continuous) and $Y$ is called a topological semigroup.

Let $X$ be a one-parameter inverse semigroup generated by the image of $P$ under a continuous homomorphism $f: P \rightarrow X$. Let $j: P \rightarrow F_{P}$ be the map $x \rightarrow(x, x, x)$. Then $j$ is an isomorphism of $P$ into $F_{P}$ and we can identify $P$ with its image in $F_{P}$. Then $x^{-1}=(1, x, 1)$ and hence $(x, y, z)=x y^{-1} z$. Furthermore, if $\bar{f}: F_{P} \rightarrow X$ is defined by

$$
\bar{f}\left(x y^{-1} z\right)=f(x) f(y)^{-1} f(z),
$$

then $\bar{f}$ is a homomorphism and the following diagram commutes [6], [1]:

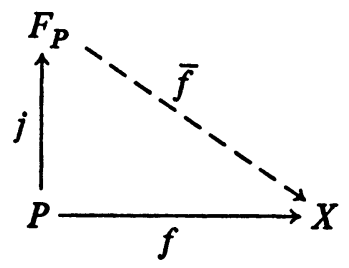

If $F_{P}$ is endowed with the relative product topology which it inherits from $R^{3}$, then the multiplication on $F_{P}$ is jointly continuous. We shall show that if $X$ is embedded densely in a compact semitopological semigroup and that if the inversion on $f(P) \cup f(P)^{-1}$ is continuous, then in fact $\bar{f}$ is a continuous homomorphism of $F_{P}$ onto $X$.

One-parameter inverse semigroups occur naturally in $B(H)$, the semigroup of bounded linear operators on Hilbert space. In the weak operator topology, the closed unit ball is a compact semitopological semigroup, and the map $T \rightarrow T^{*}$ is continuous where $T^{*}$ is the adjoint of the operator $T$. A partial isometry is an operator which is isometric on the orthogonal complement of its kernel; it is known [8, p. 99] that $T$ is a partial isometry if and only if $T T^{*} T=T$. We will show that if $J=\left\{J_{t}: t \in[0, \infty)\right\}$ is a one-parameter semigroup of partial isometries, then $J \cup J^{*}$ generates a one-parameter inverse semigroup.

This example shows that it is not unreasonable to ask that the map $x \rightarrow x^{-1}$ be continuous on $X$, and in that case we are able to give a description of $X$. 
The author is grateful to D. B. McAlister and to T. T. West for many helpful suggestions. In particular, Professor McAlister pointed out (2.2) as a generalization of (2.3).

1.1. Remark. The closure of an inverse semigroup in a compact topological semigroup is an inverse semigroup, but Brown and Moran [3, Proposition 1] give an example of a unitary group whose weak closure is not an inverse semigroup.

2. The one-parameter inverse semigroups. In this section we summarize the possible congruence relations on $F_{P}$ (and hence its homomorphic images), as given in $[6,3.10]$. We then point out that every one-parameter semigroup $J$ of partial isometries generates a one-parameter inverse semigroup $X$, and that each of the possibilities for $X$ can be realized as an inverse semigroup generated by a partial isometry.

If $1<t \in P$, let $I_{t}=\left\{x y^{-1} z: y>t\right\} \subseteq F p$ and $I_{t}^{0}=\left\{x y^{-1} z \in F_{P} ; y>\right.$ $t\} . I_{t}$ and $I_{t}^{0}$ are the only ideals of $F_{P}$. Let $\alpha$ and $\beta$ be the congruences on $F_{P}$ defined so that $x y^{-1} z \alpha r s^{-1} t$ if and only if $x=r$ and $y t=s z$ and $x y^{-1} z \beta r s^{-1} t$ if and only if $z=t$ and $y r=x s$. Then $F_{P} / \alpha \approx B p \approx F_{P} / \beta$. For each subgroup $N$ of $G$, define $\sigma_{N}$ to be the group congruence on $F_{P}$ defined so that $x y^{-1} z \sigma_{N} r s^{-1} t$ if and only if $x z s / r t y \in N$. Then if $I$ is an ideal of $F_{P}$ and $\delta=\alpha, \beta$, or $\sigma_{N}$, define the relation $I \delta$ by $x y^{-1} z I \delta r s^{-1} t$ if and only if either

(a) $x y^{-1} z$ and $r s^{-1} t$ are in $I$ and $x y^{-1} z \delta r s^{-1} t$ or

(b) $x y^{-1} z=r s^{-1} t \notin I$.

2.1. Proposition (Eberhart AND Selden). Every congruence on $F_{P}$ is $I \delta$ for some ideal $I$ and congruence $\delta=\alpha, \beta$, or $\sigma_{N}$. Hence the only possible homomorphic images of $F_{P}$ are $\left(F_{P} \backslash I\right) \cup B_{P},\left(F_{P} \backslash I\right) \cup(G / N)$ or $F_{P}[6$, Theorem 3.10].

2.2. Propostrion. Let $T$ be a semigroup with an involution ${ }^{*}$, i.e., a mapping $u \rightarrow u^{*}$ such that $(u v)^{*}=v^{*} u^{*}$ and $\left(u^{*}\right)^{*}=u$, and let $R=\left\{R_{t}: t \in P\right\}$ be a one-parameter subsemigroup of $T$. Then $\left\langle R, R^{*}\right\rangle$, the semigroup generated by $R \cup R^{*}$, is an inverse semigroup if and only if

(i) $R_{t} R_{t}^{*} R_{t}=R_{t}$ for each $t$ in $P$ and

(ii) $R_{t} R_{t}^{*} R_{s}^{*} R_{s}=R_{s}^{*} R_{s} R_{t} R_{t}^{*}$ for each $s$ and $t$ in $P$.

Proof. The conditions are clearly necessary. Now if $R$ is as above, and $s>t$, we have

$$
\begin{aligned}
R_{s} R_{t}^{*} & =R_{s-t} R_{t} R_{t}^{*}=R_{s-t} R_{s-t}^{*} R_{s-t} R_{t} R_{t}^{*} \\
& =R_{s-t} R_{t} R_{t}^{*} R_{s-t}^{*} R_{s-t}=R_{s} R_{s}^{*} R_{s-t}
\end{aligned}
$$

Similarly, $R_{t}^{*} R_{s}=R_{s-t} R_{s}^{*} R_{s}$, and it follows that any "word" from $\left\langle R, R^{*}\right\rangle$ can be expressed as a triple $R_{x} R_{y}^{*} R_{z}$ where $y>x$ and $y>z$. The arguments 
of [6, pp. 56-57] can be used to show that the map $h: F_{P} \rightarrow\left\langle R, R^{*}\right\rangle$ defined by $h\left(x y^{-1} z\right)=R_{x} R_{y}^{*} R_{z}$ is a homomorphism, and since $F_{P}$ is an inverse semigroup, so is its image $\left\langle R, R^{*}\right\rangle$.

Now let $J=\left\{J_{t}\right\}_{t=0}^{\infty}$ be a one-parameter semigroup of partial isometries on a (separable) Hilbert space. By [7, Theorem B],

$$
J_{t}=U_{t} \oplus K_{t} \oplus S_{t} \oplus T_{t},
$$

where $U_{t}$ is unitary, $K_{t}$ is purely isometric, $S_{t}$ is purely coisometric, and $T_{t}$ is a nilpotent and hence, by [12], the direct integral of truncated shifts. Recall that a truncated shift is an operator which is unitarily equivalent to $R_{t}$, where for $f$ in $\mathfrak{L}^{2}[K, a]$ (if $K$ is a separable Hilbert space and $a>0, \mathfrak{L}^{2}[K, a]$ is the Hilbert space of measurable $K$-valued functions on $[0, a]$ with squareintegrable $K$-norm):

$$
R_{b} f(x)= \begin{cases}0 & \text { if } x<t \\ f(x-t) & \text { if } t<x<a\end{cases}
$$

and $R_{t}=0$ if $t>a$. This $a$ is called the index of $R_{t}$. Since

$$
R_{t}^{*} f(x)= \begin{cases}0 & \text { if } a-t<x<a \\ f(x+t) & \text { if } 0<x<a-t\end{cases}
$$

and $R_{t}^{*}=0$ if $t>a$, we have the following.

2.3. PROPOSITION. If $R=\left\{R_{t}\right\}$ is a one-parameter semigroup of truncated shifts of index $a$, then $\left\langle R, R^{*}\right\rangle$ is isomorphic to $F_{P} / I_{a}$.

Proof. Since, for $x, y$, and $z$ in $P$, with $y<a$,

$$
R_{x} R_{y}^{*} R_{z} f(t)= \begin{cases}0 & \text { if } t<x \text { or } t>a-y+x \\ f(t-x+y-z) & \text { otherwise, }\end{cases}
$$

it is easy to see that $R$ satisfies the conditions of 2.2 and hence $\left\langle R, R^{*}\right\rangle$ is an inverse semigroup. Furthermore, one sees that $R_{x} R_{y}^{*} R_{z}=0$ if and only if $y \geqslant a$, and hence by $2.1,\left\langle R, R^{*}\right\rangle \approx F_{P} / I_{a}$.

It now follows that if $T=\left\{T_{t}\right\}$ is a one-parameter semigroup of nilpotent partial isometries of index $a$ (and hence a direct integral of truncated shifts of index less than or equal to $a)$, then $\left\langle T, T^{*}\right\rangle$ is isomorphic to $\left(F_{P} \backslash I_{a}\right) \cup\{0\}$.

Proposition 2.2 also gives the following.

2.4. Proposition. If $J=\left\{J_{t}\right\}=\left\{U_{t} \oplus K_{t} \oplus S_{t} \oplus T_{t}\right\}$ is a one-parameter semigroup of partial isometries, then $\left\langle J, J^{*}\right\rangle$ is a one-parameter inverse semigroup. Furthermore, if $\left\{U_{t}\right\} \neq I$ is a semigroup of unitary operators, $\left\{K_{t}\right\}$ of nonunitary isometries, $\left\{S_{t}\right\}$ of nonunitary coisometries, and $\left\{T_{t}\right\}$ of nilpotents of index $a$, then

(1) $\left\langle\left\{U_{t} \oplus S_{t}\right\},\left\{U_{t}^{*} \oplus S_{t}^{*}\right\}\right\rangle \approx\left(F_{P} \backslash I_{a}\right) \cup R^{+}$;

(2) $\left\langle\left\{K_{t} \oplus T_{t}\right\},\left\{K_{t}^{*} \oplus T_{t}^{*}\right\}\right\rangle \approx\left(F_{P} \backslash I_{a}\right) \cup B_{P}$ 
(3) $\left\langle\left\{K_{t} \oplus S_{t}\right\},\left\{K_{t}^{*} \oplus S_{t}^{*}\right\}\right\rangle \approx F_{P}$

(4) $\left\langle\left\{K_{t}\right\},\left\{K_{t}^{*}\right\}\right\rangle \approx B_{P}$.

3. Conditionally compact one-parameter inverse semigroups. We now assume that $X$ is a one-parameter inverse semigroup densely embedded in a compact semitopological semigroup $Y$. In case $P=\left\{1, x, x^{2}, \ldots\right\}$ where $x>1$, we show that $X$ has the discrete topology as a subspace of $Y$. Furthermore, if $P$ is dense in $[1, \infty)$, and the inversion $x \rightarrow x^{-1}$ on $P \cup P^{-1}$ is continuous, then the multiplication is continuous on $X$. We then assume, from 3.2.7 on, that inversion is continuous on $X$, and describe $\bar{X}$.

Since semigroups of the type $B_{P}$ are somewhat more accessible than those of the more general type, and some information is known about them in case $P=\left\{1, x, x^{2}, \ldots\right\}$ for $x>1$, we devote a short section to these semigroups.

3.1. Bicyclic Semigroups. As we have mentioned before, if $P=$ $\left\{1, x, x^{2}, \ldots\right\}$ where $x>1$, then $B_{P}$ is the familiar bicyclic semigroup, $B$, so-called because it is generated by two mutually inverse elements $p$ and $q$ subject to the generating relation $p q=1$; we summarize the results of [2] on this subject:

(a) $B$ is a discrete subspace of $\bar{B}$ in the relative topology. (Originally proved in [5], as was (b).)

(b) $\bar{B} \backslash B$ is a closed two-sided ideal of $\bar{B}$.

(c) The minimal idempotent $e$ of the monothetic semigroup $\Gamma=$ $\left\{q, q^{2}, \ldots\right\}$ is the minimal idempotent of $\bar{B}$, and the minimal ideal $K$ of $\Gamma$, which is a compact topological group, with identity $e$, in the relative topology from $\bar{B}$, is the minimal ideal of $\bar{B}$. Furthermore $K=e \bar{B}=\bar{B} e$.

(d) The decreasing sequence $q^{n} p^{n}$ of idempotents converges to an idempotent $w ; w B$ is a cyclic group with identity $w \bar{B}=\overline{w B}=\bar{B} \backslash B$.

(e) If the map $x \rightarrow x^{*}$ is a continuous involution, then $e$ and $w$ are selfadjoint, and for each $x \in \bar{B},(e x)^{*}=e x^{*}=(e x)^{-1}$ in $K$.

(f) If $B=\left\langle T, T^{*}\right\rangle$ where $T$ is an isometry, then $w$ is the Wold idempotent which decomposes $H$ into $H_{1} \oplus H_{2}$ where $T \mid H_{1}$ is unitary and $T \mid H_{2}$ is a unilateral shift. The minimal idempotent $e$ decomposes $H$ into $H_{3} \oplus H_{4}$, where $T \mid H_{3}$ is unitary, and $H_{4}$ is the closed linear span of the eigenvalues of $T$ (and $T^{*}$ ) corresponding to its unimodular eigenvalues.

These results show that we have no more (nor less) information about the closure of $B$ than we have about the closure of a group, which examples in [3] and [12] show may be arbitrarily pathological.

If $P$ is a dense subsemigroup of $[1, \infty)$, the existence of the idempotent $w$ follows exactly as in [2], as does the fact that $w B_{P}$ is a group. That $\bar{B}_{P} \backslash B_{P}=\overline{w B_{P}}$ turns out to be equivalent to the continuity of the inversion on $B_{P}$ as we see for $F_{P}$ in 3.2.6, and from 3.2.7 on, we shall assume this condition. 
3.2. $F_{P}$. Suppose $X=F_{P}$. Then $X$ may be pictured as below:

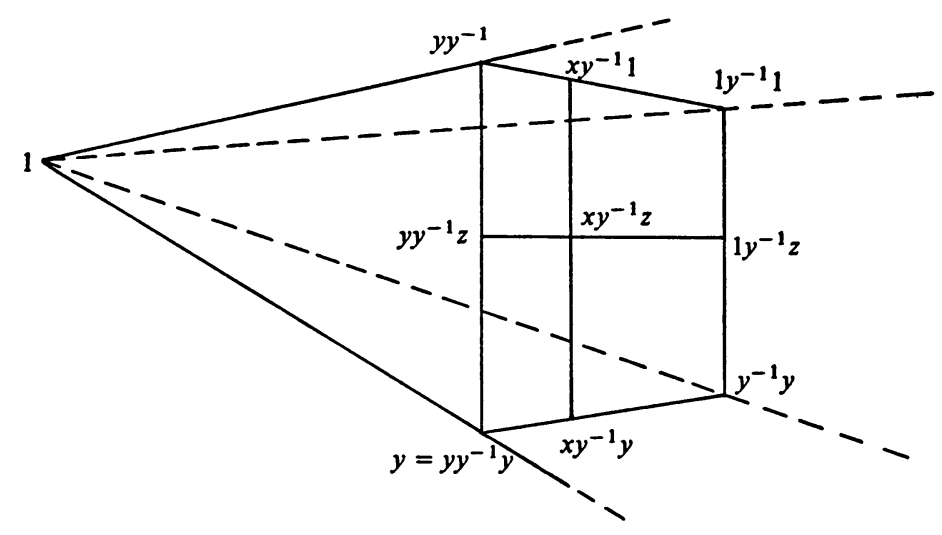

The idempotents of $F_{P}$ are precisely those of the form $x(x y)^{-1} y$ where $x$ and $y$ are elements of $P$. [6, 2.13]. Now the idempotents $\left\{x x^{-1}: x \in P\right\}$ and $\left\{x^{-1} x: x \in P\right\}$ form two decreasing nets as $x \rightarrow \infty$, and hence there exist two idempotents $E$ and $F$ such that $x x^{-1} \rightarrow E$ and $x^{-1} x \rightarrow F$ as $x \rightarrow \infty$. Since $x x^{-1}$ is decreasing, $E \neq x x^{-1}$ for any $x$ in $P$, and similarly, $F \neq x^{-1} x$ for any $x$ in $P$. Furthermore, $x x^{-1} E=E=E x x^{-1}$ for every $x$ in $P$ and $x^{-1} x F=F=F x^{-1} x$ for every $x$ in $P$.

Proposition 3.2.1. $E$ and $F$ commute with every element $x y^{-1} z$ of $F_{P}$, and hence with every element of $\bar{F}_{P}$.

PRoof. As $x \rightarrow \infty, x x^{-1} \rightarrow E$, so if $r \in P, r x x^{-1}=r\left(r^{-1} r\right)\left(x x^{-1}\right)=$ $r\left(x x^{-1}\right)\left(r^{-1} r\right)$ (since idempotents commute) $=(r x)(r x)^{-1} r \rightarrow E r$; but $r x x^{-1}$ $\rightarrow r E$, so $E r=r E$. On the other hand, for each $r$ in $P, x x^{-1} r^{-1}=$ $x x^{-1} r^{-1} r r^{-1}=r^{-1} r x x^{-1} r^{-1}=r^{-1}(r x)(r x)^{-1} \rightarrow r^{-1} E$; but $x x^{-1} r^{-1} \rightarrow E r^{-1}$, so $E r^{-1}=r^{-1} E$ for each $r$ in $P$. Hence if $x y^{-1} z \in F_{P}, E x y^{-1} z=x E y^{-1} z=$ $x y^{-1} E z=x y^{-1} z E$. Similarly, $F$ commutes with each element of $F_{P}$ and hence with each element of $\overline{F_{P}}$.

Proposition 3.2.2. If $\left\{z_{\alpha}\right\}$ and $\left\{y_{\alpha}\right\}$ are unbounded increasing nets from $P$, then $\left\{x_{\alpha} x_{\alpha}^{-1} y_{\alpha}^{-1} y_{\alpha}\right\}$ converges to $E F$.

PROOF. Let $\left\{x_{\alpha_{b}} x_{\alpha_{b}}^{-1} y_{\alpha_{b}}^{-1} y_{\alpha_{b}}\right\}$ be a subnet which converges, say to $Z$. Then

$$
E F x_{\alpha_{b}} x_{\alpha_{b}}^{-1} y_{\alpha_{b}}^{-1} y_{\alpha_{b}}=E x_{\alpha_{b}} x_{\alpha_{b}}^{-1} F y_{\alpha_{b}}^{-1} y_{\alpha_{b}}=E F \text {, }
$$

but this net converges to $E F Z$, so $E F=E F Z$. Now let $x \in P$, and assume $x_{\alpha_{8}}>x$. Then

$$
x x^{-1} x_{\alpha_{b}} x_{\alpha_{b}}^{-1} y_{\alpha_{8}}^{-1} y_{\alpha_{b}}=x_{\alpha_{b}} x_{\alpha_{b}}^{-1} y_{\alpha_{b}}^{-1} y_{\alpha_{s}} \rightarrow Z,
$$

so $x x^{-1} Z=Z$ for each $x$ in $P$. Similarly $Z x^{-1} x=Z$ for each $x$ in $P$, and 
hence, letting $x$ approach $\infty, E Z=Z=Z F$, so $E F Z=E Z F=E Z=Z$, so $E F=E F Z=Z$. It now follows that $x_{\alpha} x_{\alpha}^{-1} y_{\alpha}^{-1} y_{\alpha} \rightarrow E F$.

Proposition 3.2.3. $E F_{P} \cap F_{P}=\varnothing ; F F_{P} \cap F_{P}=\varnothing$ and $E F F_{P} \cap F_{P}=\varnothing$.

Proof. Suppose $E \in F_{P}$; then $E=x x^{-1} y^{-1} y$ for some $x$ and $y$ in $P$. But if $k>x, E=k k^{-1} E=k k^{-1} x x^{-1} y^{-1} y=k k^{-1} y^{-1} y \neq x x^{-1} y^{-1} y=E$. Thus $E$ is not in $F_{P}$, and a similar maneuver shows that $F$ and $E F$ are not in $F_{P}$. Now we show that $E F_{P} \cap F_{P}=\varnothing$. Suppose that there exists $x$ in $P$ such that $E x^{-1} \in F_{P}$. Then $E=E x x^{-1}=x E x^{-1} \in F_{P}$. So there is no such $x$. Now if $x y^{-1} z$ is any element of $F_{P}$ such that $E x y^{-1} z \in F_{P}$, then

$$
\begin{aligned}
E(y / x)^{-1} & =E x x^{-1}(y / x)^{-1}=E x y^{-1} \\
& =x y^{-1} E z z^{-1}=\left(E x y^{-1} z\right) z^{-1} \in F_{P},
\end{aligned}
$$

and this contradiction proves that there is no such $x y^{-1} z$. The proof for $F$ and $E F$ is similar.

PROPOSITION 3.2.4. If $\left\{x_{\alpha} y_{\alpha}^{-1} z_{\alpha}\right\}$ is a net from $F_{P}$ such that $\left\{y_{\alpha}\right\}$ is unbounded, then if $x_{\alpha} y_{\alpha}^{-1} z_{\alpha} \rightarrow u, u \notin F_{P}$. In fact, $u \in \overline{E F_{P}} \cup \overline{F F_{P}}$.

Proof. Note that if $e$ is any idempotent and $x$ any element of $\bar{F}_{P}$, then $e x=x$ if and only if $x \in \overline{e F_{P}}$. Now if $\left\{x_{\alpha}\right\}$ is unbounded, for any $k$ in $P, x_{\alpha}$ is eventually larger than $k$, so $k k^{-1} x_{\alpha} y_{\alpha}^{-1} z_{\alpha}=x_{\alpha} y_{\alpha}^{-1} z_{\alpha} \rightarrow u$, so $k k^{-1} u=u$, and hence $E u=u$. If $u$ were in $F_{P}, u$ would be in $E F_{P}$, so $u \notin F_{P}$. Similarly, if $\left\{z_{\alpha}\right\}$ is unbounded, $u$ would be in $F F_{P} \backslash F_{P}$. If $\left\{x_{\alpha}\right\}$ and $\left\{z_{\alpha}\right\}$ are both bounded nets and $\left\{y_{\alpha}\right\}$ is unbounded, then for every $x>\sup \left\{x_{\alpha}\right\}$,

$$
\begin{aligned}
x^{-1} x x_{\alpha} y_{\alpha}^{-1} z_{\alpha} & =x^{-1} x x_{\alpha} x_{\alpha}^{-1}\left(y_{\alpha} / x_{\alpha}\right)^{-1} z_{\alpha} \\
& =x_{\alpha} x_{\alpha}^{-1} x^{-1} x\left(y_{\alpha} / x_{\alpha}\right)^{-1} z_{\alpha} \\
& =x_{\alpha} x_{\alpha}^{-1}\left(y_{\alpha} / x_{\alpha}\right)^{-1} z_{\alpha}=x_{\alpha} y_{\alpha}^{-1} z_{\alpha} \rightarrow u .
\end{aligned}
$$

But $x^{-1} x x_{\alpha} y_{\alpha}^{-1} z_{\alpha} \rightarrow x^{-1} x u$, so $x^{-1} x u=u$, and again $u \in F F_{P} \backslash F_{P}$.

Proposition 3.2.5. If $P=\left\{1, x, x^{2}, x^{3}, \ldots\right\}$, then $F_{P}$ has the discrete topology as a subspace of any compact semitopological semigroup.

Proof. Suppose $x_{\alpha} y_{\alpha}^{-1} z_{\alpha} \rightarrow x y^{-1} z$. Then by 3.2.4, $\left\{y_{\alpha}\right\},\left\{x_{\alpha}\right\}$ and $\left\{z_{\alpha}\right\}$ are bounded nets in $P$. Hence we can find $x_{0}, y_{0}, z_{0}$ and subnets $x_{\alpha_{b}}, y_{\alpha_{s}}, z_{\alpha_{b}}$ such that $x_{\alpha_{8}} \rightarrow x_{0}, y_{\alpha_{8}} \rightarrow y_{0}$, and $z_{\alpha_{8}} \rightarrow z_{0}$. Thus eventually $x_{\alpha_{s}}=x_{0}, y_{\alpha_{8}}=y_{0}$, and $z_{\alpha_{8}}=z_{0}$, and hence eventually $x_{\alpha_{8}} y_{\alpha_{8}}^{-1} z_{\alpha_{8}}=x_{0} y_{0}^{-1} z_{0}$. Thus since

$$
x_{\alpha_{b}} y_{\alpha_{b}}^{-1} z_{\alpha_{b}} \rightarrow x y^{-1} z, \quad x_{0} y_{0}^{-1} z_{0}=x y^{-1} z \text {. }
$$

This shows that the only convergent nets are eventually constant, and so the relative topology on $F_{P}$ is the discrete.

Proposition 3.2.5 assures us that if $P$ is $\left\{1, x, x^{2}, \ldots\right\}$, then both the 
multiplication on $F_{P}$ and the inversion map on $F_{P}$ are continuous functions. Moreover, since every convergent net which is bounded in its second coordinate converges to a point of $F_{P}$, and every convergent net which is unbounded in its second coordinate converges to a point of $\overline{E P_{P}}$ or $\overline{F F_{P}}$, we see that $\overline{E F_{P}} \cup \overline{F_{P}}$ is an ideal of $\overline{F_{P}}$ and $\overline{F_{P}} \backslash F_{P}=\overline{E F_{P}} \cup \overline{F F_{P}}$. We next show that all these related conditions are equivalent.

Proposition 3.2.6. If $F_{P}$ is contained densely in a compact semitopological semigroup, and $P \subseteq F_{P}$ is a continuous embedding, then the following are equivalent:

(a) Inversion is continuous on $P \cup P^{-1}$;

(b) $P^{-1} \subseteq F_{P}$ is a continuous embedding;

(c) The topology on $F_{P}$ is the product topology;

that is, $x_{\alpha} y_{\alpha}^{-1} z_{\alpha} \rightarrow x y^{-1} z$ if and only if $x_{\alpha} \rightarrow x, y_{\alpha} \rightarrow y$, and $z_{\alpha} \rightarrow z$;

(d) If $\left\{y_{\alpha}\right\}$ is a bounded net from $P$, and if $x_{\alpha} y_{\alpha}^{-1} z_{\alpha} \rightarrow u, u \in F_{P}$;

(e) $\bar{F}_{P} \backslash F_{P}=\overline{E F_{P}} \cup \overline{F F_{P}}$.

(f) $\bar{F}_{P} \backslash F_{P}$ is an ideal of $F_{P}$, if $\bar{F}_{P} \backslash F_{P} \neq \varnothing$.

Proof. The implications (a) $\Leftrightarrow$ (b) and (c) $\Rightarrow$ (d) are easy. We show (a) $\Rightarrow$ (c) and (d) $\Rightarrow($ a), and (d) $\Rightarrow(e) \Rightarrow(f) \Rightarrow(d)$.

(a) $\Rightarrow$ (c). Suppose inversion is continuous in $P \cup P^{-1}$. We note that if $x_{\alpha} y_{\alpha}^{-1} z_{\alpha} \rightarrow u$ where $x_{\alpha} \rightarrow x$ and $z_{\alpha} \rightarrow z$, and if $x>1$, and $1<r<x$, then since $r<x_{\alpha}$ eventually, $r r^{-1} x_{\alpha} y_{\alpha}^{-1} z_{\alpha}=x_{\alpha} y_{\alpha}^{-1} z_{\alpha}$ eventually, so $r r^{-1} u=u$. (Similarly $u t^{-1} t=u$ if $t<z$.) Now if $x_{\alpha} \rightarrow x$, we show that $x_{\alpha} x_{\alpha}^{-1} \rightarrow x x^{-1}$. We can assume that $x_{\alpha} x_{\alpha}^{-1} \rightarrow u$ for some $u \in \bar{F}_{p}$. If $r>x$, then eventually $r>x_{\alpha}$, so $r^{-1} x_{\alpha} x_{\alpha}^{-1}=r^{-1}$, and so $r^{-1}=r^{-1} u$; letting $r \rightarrow x, r^{-1} \rightarrow x^{-1}$ by assumption and thus $x^{-1}=x^{-1} u$, and $x x^{-1}=x x^{-1} u$. If $x_{\alpha} \geqslant x$ frequently, then

$$
x x^{-1} x_{\alpha} x_{\alpha}^{-1}=x_{\alpha} x_{\alpha}^{-1}
$$

frequently, so $x x^{-1} u=u$. On the other hand, if $x_{\alpha}<x$ eventually, then since $x x_{\alpha}^{-1} \rightarrow x x^{-1}$ we have

$$
x x_{\alpha}^{-1} u=\left(x / x_{\alpha}\right) x_{\alpha} x_{\alpha}^{-1} u=x / x_{\alpha} u
$$

by the above, so $x x^{-1} u=u$, so in either case, $u=x x^{-1} u=x x^{-1}$. Similarly $x_{\alpha}^{-1} x_{\alpha} \rightarrow x^{-1} x$. Now if $x_{\alpha} \rightarrow x, y_{\alpha} \rightarrow y, z_{\alpha} \rightarrow z$, we can assume $x_{\alpha} y_{\alpha}^{-1} z_{\alpha} \rightarrow u$. Let $r>x$ and $t>z$; then

$$
r r^{-1} x_{\alpha} y_{\alpha}^{-1} z_{\alpha} t^{-1} t=r\left(\frac{r_{\alpha} t}{x_{\alpha} z_{\alpha}}\right)^{-1} t,
$$

so

$$
r r^{-1} u t^{-1} t=r\left(\frac{r y t}{x z}\right)^{-1} t=r r^{-1}(y t / x z) t .
$$


Letting $r \rightarrow x$, we have $x x^{-1} u t^{-1} t=x x^{-1}(y t / x z)^{-1} z=x(y t / z)^{-1} t=$ $x(y / z)^{-1} t^{-1} t$, and letting $t \rightarrow z$,

$$
u=x x^{-1} u z^{-1} z=x(y / z)^{-1} z^{-1} z=x y^{-1} z .
$$

It follows that $x_{\alpha} y_{\alpha}^{-1} z_{\alpha} \rightarrow x y^{-1} z$ if and only if $x_{\alpha} \rightarrow x, y_{\alpha} \rightarrow y$, and $z_{\alpha} \rightarrow z$.

(d) $\Rightarrow$ (a). Let $x_{\alpha} \rightarrow x$ in $P$, and suppose $x_{\alpha}^{-1} \rightarrow r s^{-1} t$ in $F_{P}$. We show $r \leqslant x$, and $t \leqslant x$. For suppose $r>x$. Then $r>x_{\alpha}$ eventually, so since

$$
r r^{-1}\left(\frac{r}{x_{\alpha}}\right)=r x_{\alpha}^{-1} \rightarrow r r s^{-1} t=r^{2}\left(\frac{r^{2} s}{r^{2} \wedge s}\right) \frac{r^{2} t}{r^{2} t}
$$

we have

$$
r r^{-1} r / x=r^{2}\left(\frac{r^{2} s}{r^{2} \wedge s}\right)^{-1} \frac{r^{2} t}{r^{2} \wedge s}
$$

and hence $r=1$, and this contradiction shows that $r \leqslant x$; similarly $t \leqslant x$. Now suppose that $s<x$. Then since $s<x_{\alpha}$ eventually, and $x_{\alpha}^{-1}=x_{\alpha}^{-1} s s^{-1}$ $\rightarrow r s^{-1} t s s^{-1}=r t(s t)^{-1} t$, we have $t=1$. Similarly, we can show $r=1$. Thus $x_{\alpha}^{-1} \rightarrow s^{-1}$ where $s<x$. Now if $x_{\alpha}<x$ frequently, the fact that

$$
(x / x) x^{-1}=x_{\alpha}^{-1} x x^{-1} \rightarrow s^{-1} x x^{-1}=(x / s) x^{-1}
$$

implies that $x=s$. If $x_{\alpha}>x$ frequently, then

$$
s^{-1}=\lim _{\alpha} x_{\alpha}^{-1}=\lim _{\alpha}\left(x_{\alpha}^{-1} x x^{-1}\right)=s^{-1} x x^{-1}=(x / s) x^{-1},
$$

so $x=s$. Hence if $x_{\alpha}^{-1} \rightarrow r s^{-1} t, r \leqslant x, t \leqslant x$, and $s>x$. Suppose $s>x$. Then $s>x_{\alpha}$ eventually, so since $\left(s / x_{\alpha}\right) s^{-1}=x_{\alpha}^{-1} s s^{-1} \rightarrow r s^{-1} t s s^{-1}=$ $r t(s t)^{-1} t, t=1$, and $s / x=r$ Similarly, $r=1$, so $s=x$. Therefore, if $x_{\alpha} \rightarrow x$, then $x_{\alpha}^{-1} \rightarrow x^{-1}$.

(d) $\Rightarrow$ (e). Let $u \in \bar{F}_{P} \backslash F_{P}$. Then $u=\lim _{\alpha} x_{\alpha} y_{\alpha}^{-1} z_{\alpha}$, where $\left\{y_{\alpha}\right\}$ is unbounded. As in 3.2.4, either $E u=u$ or $F u=u$. Hence $u \in \overline{E F}_{P} \cup \overline{F F}_{P}$. If $v \in \overline{E F}_{P} \cup \overline{F F}_{P}$, then $E v=v$ or $F v=v$, so by 3.2.3, $v \in \bar{F}_{P} \backslash F_{P}$.

(e) $\Rightarrow$ (f). Suppose $\bar{F}_{P} \backslash F_{P}=\overline{E F}_{P} \cup \overline{F F}_{P}$. If $u \in \bar{F}_{P} \backslash F_{P}$, then either $E u=$ $u$ or $F u=u$, so if $v \in \bar{F}_{P}, u v=E u v \in \overline{E F}_{P}$, or else $u v=F u v \in \overline{F F}_{P}$, so $u v \in \bar{F}_{P} \backslash F_{P}$.

$(\mathrm{f}) \Rightarrow(\mathrm{d})$. We first show that if $\left\{y_{\alpha}\right\}$ is bounded and $y_{\alpha}^{-1} \rightarrow u$, then $u \in F_{P}$. Let $r>y_{\alpha}$ for each $\alpha$. We can assume $y_{\alpha}$ converges, say to $y$ in $P$, and hence $r^{-1} r u=r^{-1} r / y$, and since $r^{-1} r u \in F_{P}, u \in F_{P}$. Now let $x_{\alpha} y_{\alpha}^{-1} z_{\alpha} \rightarrow v$. Let $r>x_{\alpha}$ and $t>z_{\alpha}$ for each $\alpha$. Then

$$
r r^{-1} x_{\alpha} y_{\alpha}^{-1} z_{\alpha} t^{-1} t=r\left(\frac{r_{\alpha} t}{x_{\alpha} z_{\alpha}}\right)^{-1} t
$$

we can assume $r y_{\alpha} t^{-1} / x_{\alpha} z_{\alpha}$ converges to $q$, hence $q \in F_{P}$; so $r r^{-1} v t^{-1} t=r q t$ $\in F_{P}$ and hence $v \in F_{P}$. 
3.3. For the remainder of this paper, we will assume $X$ is a homomorphic image of $F_{P}$ embedded densely in a compact semitopological semigroup, and that the inversion on $X$ is a continuous mapping.

Proposition 3.3.1. If $X=F_{P} / \delta$ and if $\delta$ is the natural map of $F_{P}$ to $X$, then, under the assumption that inversion is continuous on $X, \delta^{\natural}$ is a continuous homomorphism from $F_{P}$ with the product topology onto $X$.

Proof. It follows as in 3.2.6 that $\delta^{\natural}$ is continuous on $F_{P} \backslash I$ where $\delta=I \sigma$. Now since $\delta^{\natural}$ must be continuous on $P$, we know $I=I_{a}$ for some $a>1$ and if $\sigma=\sigma_{N}$ for some subgroup $N$ of $G, N$ must be a closed subgroup of $G$. We can assume $P$ is dense in $[1, \infty)$. We prove the proposition for $F_{P} / I_{a} \alpha$ and point out that the proofs for the remaining cases are similar.

Suppose $X=F_{P} / I_{a} \alpha$. Then we note that $X \approx\left(F_{P} \backslash I_{a}\right) \cup B_{P}$, where the multiplication is as follows: If both $u$ and $v$ are in $F_{P}$ then $u v$ is as usual. If either $u$ or $v$ is in $B_{P}$, then $u v=\alpha^{\xi}(u) \alpha^{\xi}(v)$. For the sake of simplifying the notation, we equate $x y^{-1} z$ with $(x, y / z)$ if $y>a$.

Let $x_{\alpha} \rightarrow a$ from $[1, a)$, and suppose $x_{\alpha} x_{\alpha}^{-1} \rightarrow e$. Since for each $r<a$, $x_{\alpha} x_{\alpha}^{-1} r r^{-1}=x_{\alpha} x_{\alpha}^{-1}$ eventually, err ${ }^{-1}=e$. Now $x_{\alpha} x_{\alpha}^{-1}(a, a)=(a, a)$, so $e(a, a)=(a, a)$. But $e\left(a / x_{\alpha}\right)^{-1} \rightarrow e$ as $x_{\alpha} \rightarrow a$, and

$$
e\left(a / x_{\alpha}\right)^{-1}=e x_{\alpha} x_{\alpha}^{-1}\left(a / x_{\alpha}\right)^{-1}=e\left(x_{\alpha}, a\right) \rightarrow e(a, a)=(a, a),
$$

so $e=(a, a)$. Similarly, if $x_{\alpha}^{-1} x_{\alpha} \rightarrow f, f(1,1)=(1,1)$, so since

$$
f\left(a / x_{\alpha}\right)=f x_{\alpha}^{-1} x_{\alpha}\left(a / x_{\alpha}\right)=f x_{\alpha}^{-1}(a, 1)=f\left(a / x_{\alpha}, 1\right) \rightarrow f(1,1)
$$

and $f\left(a / x_{\alpha}\right) \rightarrow f$, we have $f=f(1,1)=(1,1)$. Now suppose that $x_{\alpha} \rightarrow x$, and $y_{\alpha} \rightarrow b>a$; we assume $x_{\alpha} y_{\alpha}^{-1} \rightarrow u$. Then $y_{\alpha}>a$ eventually, so $x_{\alpha} y^{-1}=$ $\left(x_{\alpha}, y_{\alpha}\right)$ eventually, and $x_{\alpha} y_{\alpha}^{-1} \rightarrow(x, b)$. If $y_{\alpha} \rightarrow a$, we can assume $y_{\alpha}<a$ eventually. As above, if $r\left\langle a, x_{\alpha} y_{\alpha}^{-1}=x_{\alpha} y_{\alpha}^{-1} r r^{-1} \rightarrow u r r^{-1}\right.$ so $u=u r r^{-1}$ and hence, letting $r \rightarrow a, u=u(a, a)$. But $x_{\alpha} y_{\alpha}^{-1}(a, a) \rightarrow u(a, a)$ and $x_{\alpha} y_{\alpha}^{-1}(a, a)$ $=\left(x_{\alpha} a / y_{\alpha}, a\right) \rightarrow(x, a)$ so $u=(x, a)$. If $z_{\alpha} \rightarrow z$, and $y_{\alpha} \rightarrow b>a$, then since $y_{\alpha}^{-1} z_{\alpha}=\left(1, y_{\alpha} / z_{\alpha}\right)$ eventually, $y_{\alpha}^{-1} z_{\alpha} \rightarrow(1, b / z)$. If $y_{\alpha} \rightarrow a$, assume $y_{\alpha}^{-1} z_{\alpha} \rightarrow u$, and $y_{\alpha}<a$ eventually. Then if $r<a, r^{-1} r u=u$, and, letting $r \rightarrow a,(1,1) u=$ $u$ by the above; but $(1,1)\left(y_{\alpha}^{-1} z_{\alpha}\right)=\left(1, y_{\alpha} / z_{\alpha}\right) \rightarrow(1, a / z)$ so $u=(1, a / z)$. Now we show in general that if $x_{\alpha} \rightarrow x, y_{\alpha} \rightarrow y$, and $z_{\alpha} \rightarrow z$, then $x_{\alpha} y_{\alpha}^{-1} z_{\alpha} \rightarrow$ $x y^{-1} z$. We can assume $x_{\alpha} y_{\alpha}^{-1} z_{\alpha} \rightarrow u$. If $y>a$, then $x_{\alpha} y_{\alpha}^{-1} z_{\alpha}=\left(x_{\alpha}, y_{\alpha} / z_{\alpha}\right)$ eventually and we are done. If $y=a$ and $x>1$, suppose $x<a$, and let $x<r<a$. Then eventually $r r^{-1} x_{\alpha} y_{\alpha}^{-1} z_{\alpha}=r\left(r y_{\alpha} / x_{\alpha}\right)^{-1} z_{\alpha} \rightarrow(r, r y / x z)$ so $r r^{-1} u=(r, r y / x z)$, and, letting $r \rightarrow x, x x^{-1} u=(x, y / z)$ but as before $x x^{-1} u$ $=u$, so $u=(x, y / z)$. Finally, if $x=a$, then $u=(a, a) u=(a, a / z)$. Thus if $\delta^{\xi}\left(x_{\alpha}\right) \rightarrow \delta^{\natural}(x), \delta^{\dagger}\left(y_{\alpha}\right) \rightarrow \delta^{\natural}(y)$, and $\delta^{\dot{\zeta}}\left(z_{\alpha}\right) \rightarrow \delta^{\natural}(z)$, then $\delta^{\natural}\left(x_{\alpha} y_{\alpha}^{-1} z_{\alpha}\right) \rightarrow$ $\delta^{y}\left(x y^{-1} z\right)$, and it follows that $\delta^{\natural}$ is a continuous homomorphism of $F_{P}$ onto $X$. The proof is exactly analogous if $X=F_{P} / I_{a} \sigma_{N}$. 
Now recall that for each $X$, the decreasing nets $\left\{\delta^{\natural}(x) \delta^{\natural}(x)^{-1}: x \in P\right\}$ and $\left\{\delta^{y}(x)^{-1} \delta^{\phi}(x): x \in P\right\}$ converge to idempotents $E$ and $F$ respectively as $x \rightarrow \infty$. Furthermore, in each case, $E$ and $F$ commute with $X$, and 3.3.1 allows us to conclude that in fact, $\bar{X}=X \cup \overline{E X} \cup \overline{F X}$. We point out that if $X=F_{P} / I_{a} \sigma_{N}, E=F=\delta^{\zeta}\left(a a^{-1}\right)$ is the identity element of the group which is the minimal ideal of $X$. If $X=F_{P} / I_{a} \alpha, F=(1,1)=\delta^{\phi}\left(a^{-1} a\right)$ but $E \notin X$. In this case, $\bar{X} \backslash X=\overline{E X}$. Similarly, if $X=F_{P} / I_{a} \beta$, then $\bar{X} \backslash X=\overline{F x}$.

Proposition 3.3.3. If $X$ is any homomorphic image of $F_{P}$, then $E X \approx B_{P}$ or $E X$ is a commutative group; $E X$ is a group if and only if $E=E F$, and these remarks are true if $E$ and $F$ are interchanged. Furthermore, $\bar{X}=X$ $\cup \overline{E X} \cup \overline{F X}$.

Proof. Since $E$ commutes with each element of $X$, the map $u \rightarrow E u$ is a homomorphism of $X$ to $E X$. Hence $E X=F_{P} / I_{b} \sigma$ for some $b \in P$ and congruence $\sigma$ on $F P$. Since $E x x^{-1}=E$ for each $x$ in $\left(I_{b} \sigma\right)^{\phi}(P), \mathrm{b}=1$, and hence $E X=F_{P} / \beta$ or $F_{P} / \sigma_{N}$. In the first case, $E X \approx B_{P}$ with $E$ as identity and

$$
E \delta^{\xi}\left(x y^{-1} z\right)=E(y / x)^{-1} z \approx(y / x, z),
$$

and the idempotents $E x^{-1} x$ converge to $E F$ as $x \rightarrow \infty$. It is easy to see that $E F X$ is a commutative group in this case. In the second case, $E X$ is a group and since $E x^{-1} x=E, E=E F$. The discussion in the previous paragraph together with 3.2.6 implies that $\bar{X}=X \cup \overline{E X} \cup \overline{F X}$.

The idempotents $E$ and $F$ are thus maximal among idempotents of $\bar{X} \backslash X$, if $\bar{X} \backslash X \neq \varnothing$. We now turn to a discussion of minimal idempotents of $\bar{X}$. The one-parameter semigroups $\overline{\delta^{\xi}(P)}$ and $\overline{\delta^{\xi}\left(P^{-1}\right)}$ possess as kernels (minimal ideals) commutative compact topological groups $K$ and $K^{\prime}$, respectively, with identities $e_{0}$ and $f_{0}$. If $X=B_{P}$, it follows as in [2] that $e_{0}=f_{0}$ and hence $K=K^{\prime}$ is the minimal ideal for $\bar{X}$; furthermore $K \subseteq \overline{E X}$ and $K=\overline{E X}$ if and only if $e_{0}=E=w$. These facts are easy to prove if $X$ is any of the other images of $F_{P}$; we discuss the case where $X=F_{P}$.

If $\left\{x_{\alpha}\right\}$ is a net from $P$ which converges to $e_{0}$, by 3.2.6, $\left\{x_{\alpha}\right\}$ is unbounded. If $x \in P, x<x_{\alpha}$ frequently, so $x x^{-1} x_{\alpha}=x_{\alpha}$ frequently and hence $x x^{-1} e_{0}=$ $e_{0}=E e_{0}=e_{0} E$. Similarly $e_{0} F=e_{0}=F e_{0}$. Thus $E F e_{0}=e_{0}$ so $e_{0} \in \overline{E F X}$, and hence $e_{0}$ commutes with each element of $\bar{X}$. Now if $y \in P, x_{\alpha} y^{-1} \rightarrow e_{0} y^{-1}$. But since $x_{\alpha}>y$ frequently, $x_{\alpha} y^{-1}=x_{\alpha} x_{\alpha}^{-1} x_{\alpha} / y$, and $e_{0} x_{\alpha} y^{-1} \rightarrow e_{0} y^{-1}$. Thus since $e_{0} x_{\alpha} y^{-1}=e_{0} x_{\alpha} x_{\alpha}^{-1} x_{\alpha} / y=e_{0} x_{\alpha} / y, e_{0} y^{-1}$ is in $\bar{K}=K$. Similarly $y^{-1} e_{0}$ is in $K$. Thus $e_{0} X=X e_{0} \subseteq K$, and $e_{0} \bar{X}=\bar{X} e_{0} \subseteq K$. Now if $e$ is any idempotent of $\bar{X},\left(e_{0} e\right)\left(e_{0} e\right)=e_{0} e_{0} e e=e_{0} e$, and since $e_{0} e \in K, e_{0} e=e$. Hence $e_{0}$ is the minimal idempotent for $\bar{X}$. It follows that $e_{0}=f_{0}$ and as in [2, Proposition 2] that $K$ is the kernel for $\bar{X}$ and that $K=e_{0} \bar{X}$. We summarize this discussion in the following proposition. 
Proposition 3.3.4. If $X=F_{P} / \delta$, then the kernel of $\bar{X}$ is $K$, the compact topological group which is the kernel of $\overline{\delta^{\xi}(P)}$. If $e_{0}$ is the identity element of $K$, then $K=e_{0} \bar{X}$.

We now derive some corollaries and give some examples from the space of bounded linear operators on complex Hilbert space. This one is proved as in [2, Proposition 5].

COROLlary 3.3.5. If $\bar{X}$ is a compact semitopological semigroup with a continuous involution $t \rightarrow t^{*}$, all idempotents of $X, E X$ and $F X$ are selfadjoint, as are $E F$ and $e_{0}$. If $x \in K$, then $x^{*} \in K$, and furthermore, $x^{*} x=e=x x^{*}$ for every $x$ in $K$.

The following corollary was proved in [1, Theorem 4].

COROLlaRY 3.3.6. If $\bar{X}$ is a compact topological semigroup, then since $E X \neq B_{P} \neq F X, E=F$ and hence $\bar{X}=X \cup \overline{E X}$.

EXAMPLE 3.3.7. If $U$ is unitary, and $T$ and $S$ are unilateral shifts, let $J=U \oplus T \oplus S^{*}$. Then $J^{n}=U^{n} \oplus T^{n} \oplus\left(S^{*}\right)^{n}$; let $X=\left\langle J, J^{*}\right\rangle$. We have $E=I_{1} \oplus O_{2} \oplus I_{3}, F=I_{1} \oplus I_{2} \oplus O_{3}$, and $E F=I_{1} \oplus O_{2} \oplus O_{3}$. EX is the bicyclic semigroup generated by $U \oplus O_{2} \oplus S^{*}=p$ and $U^{*} \oplus O_{2} \oplus S=q$ with $1=p q=E$, and $F X$ is the bicyclic semigroup $\left\langle U \oplus T \oplus O_{3}, U^{*} \oplus T^{*}\right.$ $\oplus O_{3}>$. It is easy to see that the structure of $\overline{E F X}$ is totally dependent on $U$.

REMARK 3.3.9. If $\left\{J_{t}\right\}=J$ is a one-parameter semigroup of partial isometries on a finite-dimensional complex space, then $J_{t}=U_{t} \oplus T_{t}$, where $U_{t}$ is unitary and $T_{t}$ is a truncated shift.

ProOF. The uniform and weak topologies coincide, so $\bar{X}=\left\langle J \cup J^{*}\right\rangle$ is a compact topological semigroup. If $J_{t}=U_{t} \oplus S_{t} \oplus C_{t}^{*} \oplus T_{t}$ where either $S_{t}$ or $C_{t}$ is a nonunitary isometry, then either $E X$ or $F X$ would be bicyclic. Since this cannot happen in a compact topological semigroup, $S_{t}$ and $C_{t}^{*}$ are both unitary.

\section{REFERENCES}

1. M. Bertman, Free topological inverse semigroups, Semigroup Forum 8 (1974), 226-270.

2. M. Bertman and T. T. West, Conditionally compact bicyclic semitopological semigroups, Proc. Roy. Irish Acad. Sect. A 76 (1975), 219-226.

3. G. Brown and W. Moran, Idempotents of compact monothetic semigroups, Proc. London Math. Soc. 22 (1971), 203-216.

4. A. H. Clifford and G. Preston, The algebraic theory of semigroups, vol. I, Math. Surveys, no. 7, Amer. Math. Soc., Providence, R.I., 1961.

5. C. Eberhart and J. Selden, On the closure of the bicyclic semigroup, Trans. Amer. Math. Soc. 144 (1969), 115-126.

6. One-parameter inverse semigroups, Trans. Amer. Math. Soc. 168 (1972), 53-66.

7. M. Embry, A. Lambert and L. Wallen, A simplified treatment of the structure of semigroups of partial isometries, Michigan Math. J. 22 (1975), 175-179.

8. P. R. Halmos, A Hilbert space problem book, Van Nostrand, Princeton, N. J., 1967. 
9. K. H. Hofmann and P. S. Mostert, Elements of compact semigroups, Merrill, Columbus, Ohio, 1966.

10. K. DeLeeuw and I. Glicksberg, Applications of almost periodic compactifications, Acta Math. 105 (1961), 63-98.

11. D. B. McAllister, A homomorphism theorem for semigroups, J. London Math. Soc. 43 (1968), 355-366.

12. L. J. Wallen, Decomposition of semi-groups of partial isometries, Indiana Univ. Math. J. 20 (1970), 207-212.

13. T. T. West, Weakly compact monothetic semigroups of operators in Banach spaces, Proc. Roy. Irish Acad. Sect. A 67 (1968), 27-37.

Department of Mathematics, Clarkson College, Potsdam, New York 13676 